\title{
Si-Miao-Yong-An Decoction maintains the cardiac function and protects cardiomyocytes from myocardial ischemia and reperfusion injury
}

wenwen cui

Hebei University of Chinese Medicine https://orcid.org/0000-0003-0391-4150

\section{Xin Shen}

Chinese Academy of Medical Sciences \& Peking Union Medical College Institute of Biomedical

Engineering

Lingjuan Zhu

Shenyang Pharmaceutical University

Mingye Wang

Hebei University of Chinese Medicine

\section{Yuanyuan Hao}

Hebei University of Chinese Medicine

\section{Yuqian Zhao}

Tsinghua University

Yang Li ( $\square$ doctoryangli@hrbmu.edu.cn )

Yunlong Hou

Hebei University of Chinese Medicine

\section{Research}

Keywords: Si-Miao-Yong-An Decoction, myocardial infarction, myocardial ischemia/reperfusion injury, autophagy activation, anti-pyroptosis

Posted Date: May 10th, 2021

DOI: https://doi.org/10.21203/rs.3.rs-476965/v1

License: (c) (i) This work is licensed under a Creative Commons Attribution 4.0 International License.

Read Full License 


\section{Si-Miao-Yong-An Decoction maintains the cardiac function and protects cardiomyocytes from myocardial ischemia and reperfusion \\ injury}

Wenwen Cui ${ }^{1 \#}$, Xin Shen ${ }^{2 \#}$, Lingjuan $\mathrm{Zhu}^{3}$, Mingye Wang ${ }^{1}$, Yuanyuan Hao ${ }^{1}$, Yuqian $\mathrm{Zhao}^{4}$, Yang $\mathrm{Li}^{5^{*}}$, Yunlong $\mathrm{Hou}^{1 *}$

1. College of Integrated Chinese and Western Medicine, Hebei University of Chinese Medicine, Shijiazhuang,050035, China

2. Department of Pharmacy, National Cancer Centre / Cancer Hospital, Chinese Academy of Medical Sciences and Peking Union Medical College, Beijing 100021, China

3. School of Traditional Chinese Materia Medica, Key Laboratory of Structure-Based Drug Design \& Discovery of Ministry of Education, Shenyang Pharmaceutical University, Shenyang, 110016, China.

4.School of Life Science, Tsinghua University, Beijing, 100084, China.

5. Department of cardiology, The fourth affiliated hospital of Harbin Medical University / The Heilongjiang Academy of Medical Science, Harbin, 150001, China

\# Both authors contribute equally.

${ }^{*}$ Corresponding authors.

E-mail addresses: reaishenghuo0506@163.com(W.Cui),doctoryangli@hrbmu.edu.cn (Y. Li), houyunlonghrb@Hotmail.com (Y. Hou). 


\begin{abstract}
Objective : The aim of this study was to determine whether Si-Miao-Yong-An decoction (SMYAD) could protect cardiomyocytes from ischemia/reperfusion (I/R) injury and its underlying mechanisms.

Methods: C57BL/6 mice were used to establish a model of myocardial infarction by $\mathrm{I} / \mathrm{R}$ injury and treated by SMYAD for 4 weeks. Then the cardiac functions of mice were evaluated by Cardiac Magnetic Resonance (CMR). Histopathological analysis for the heart remodeling was detected by H\&E and Masson staining. The protein expression of Collagen I, MMP9, and TNF $\alpha$ was detected by Western blot in the heart tissues. H9C2 cells were used to establish the hypoxia/reoxygenation $(\mathrm{H} / \mathrm{R})$ model and SMYAD intervention. MTT assays detected the cell viability of myocardial cells. The expression level of IL-1 $\beta$ was evaluated by ELISA. The expression levels of LC3B-II/LC3B-I, pmTOR, mTOR, NLRP3, pro-Caspase1, and cleaved-Caspase1 in H9C2 cells were evaluated by Western blot.
\end{abstract}

Results: SMYAD improved cardiac functions such as ventricular volume and ejection fraction of the rats with ischemia/reperfusion injury. Morphological assay indicated that SMYAD reduced the scar size and inhibited fibrosis formation. It was found that SMYAD could regulate Collagen I, MMP9, and TNF $\alpha$ protein expression levels in the heart tissues. SMYAD improved the survival rate of cardiomyocytes $\mathrm{H} 92 \mathrm{C}$ in the $\mathrm{H} / \mathrm{R}$ injury model. SMYAD elevated the rate of LC3B-II/LC3B-I protein expression, decreased the rate of $\mathrm{p}-\mathrm{mTOR} / \mathrm{mTOR}$ protein expression, and reduced expressions of Caspase 1, NLRP3, and IL-1 $\beta$ in H/R cardiomyocytes.

Conclusion: SMYAD exerted protective effects on ischemia-reperfusion injury in myocardial cells by activating autophagy and inhibiting pyroptosis. This might be the reason why SMYAD protected myocardial tissue and improved cardiac function in mice with ischemia/reperfusion.

Keywords : Si-Miao-Yong-An Decoction; myocardial infarction; myocardial ischemia/reperfusion injury; autophagy activation; anti-pyroptosis 


\section{INTRODUCTION}

Myocardial infarction (MI) is an event of myocardial ischemia caused by coronary artery occlusion and interruption of blood flow leading to myocardial cell death[1], which retains a significant impact on global health and economic burden [2, 3]. Although timely reperfusion has proven to be the most effective treatment, the following ischemia/reperfusion (IR) injury indeed undermines the effectiveness and presented potential risks. All efforts to seek ways and means of protecting cardiomyocytes from IR injuries are necessarily exerted with a pressing sense of urgency.

Programmed cell death (PCD) is a gene-directed way of cell self-death for normal cell turnover and tissue homeostasis, including apoptosis, autophagy, necroptosis and pyroptosis, which occurs during the normal development of individuals and in abnormal physiological states or diseases [4-6]. The consensus among the pathological mechanisms of $\mathrm{I} / \mathrm{R}$ injury is the programmed cell death of cardiomyocytes caused by histanoxia [2]. When myocardial I/R injury occurs, the increase of oxygen free radicals and calcium overload lead to massive death of myocardial cells and release endogenous danger molecules, damage-associated molecular patterns (DAMPs), which will trigger and recruit abundant of inflammatory cells to clear cell debris away, in turn, undue inflammation aggravates the heart injury[7, 8]. Along with researches going deep, DAMPs released from host cells can initiate and assemble cytosolic multiprotein oligomers called inflammasomes in immune cells such as macrophages. Initiation of the inflammasomes promotes cleavage and secretion of pro-inflammatory cytokines IL$1 \beta$ and IL-18, as well as cleavage of Gasdermin-D, which induces a distinct form of programmed cell death, referred to as pyroptosis [9-11]. Experimental evidence also suggests that pyroptosis is a primary mechanism to aggregate the cardiomyocyte death in the myocardial I/R injury [12]. Thus, the inhibition of pyroptosis holds promise as a therapeutic target for myocardial ischemia/reperfusion injury [13, 14]. In addition to the assembly of inflammasomes in the process of pyroptosis, the appearance of autophagosomes is also associated with PCD, referred to as autophagy. Recent researches have revealed that the activation of autophagy accompanied with PCD is an endogenic survival mechanism $[15,16]$. In a sense, autophagy might counterbalance and regulate PCD to maintain the cell viability. There is a reasonable prospect that a therapeutic approach activating autophagy and inhibiting pyroptosis will be promising in protecting myocardiocytes from $\mathrm{I} / \mathrm{R}$ injury.

Si-Miao-Yong-An decoction (SMYAD) is a traditional Chinese medicine formulation, which consists of Lonicerae Japonicae Flos (Jinyinhua), Scrophulariae Radix (Xuanshen), Angelica Sinensis Radix (Danggui), and Glycyrrhizae Radix et Rhizoma (Gancao) in a 3:3:2:1 proportion by weight. SMYAD first appeared in the "Hua Tuo Shen Yi Mi Zhuan" of the Eastern Han Dynasty and was edited in the "Yan Fang Xin Bian" of the Qing Dynasty. SMYAD was also listed as one of the 100 classic prescriptions in the catalog of the "Catalogue of Ancient Classic Prescriptions (the First Batch)", which was issued by the National Administration of Traditional Chinese Medicine in 2018. This famous ancient recipe was traditionally used for gangrene and 
in modern medicine to treat peripheral vascular diseases, diabetes, coronary heart disease, and heart failure[17]. According to reports, SMYAD has been reported to exert pharmacological effects, including anti-inflammatory, regulating angiogenesis, antioxidative stress, regulating blood lipids, and improving blood rheology[18-20]. However, the effect of SMYAD in myocardial I/R injury has yet not been explored.

In this study, we performed in vivo $\mathrm{I} / \mathrm{R}$ injury mouse model to prove the protective effects of SMYAD on myocardial injuries and carried out studies on the mechanism of actions in vitro. The main objective of this study is to investigate the pharmacological effects of SMYAD on MI and provide the experimental basis for clinical application.

\section{MATERIALS AND METHODS}

\section{Materials}

Sodium chloride injection (Harbin Sanlian Pharmaceutical, China);Dulbecco's modified Eagle's medium (DMEM) (Gibco, American); Fetal bovine serum (Gibco, American); penicillin/streptomycin (Gibco, American); 0.25\% (w/v) Trypsin-0.53mM EDTA (Gibco, American); glucose- free DMEM (Gibco, American); IL-1 $\beta$ ELISA Kit (Elabscience, China); BCA Assay Kit (TDY Biotechnology Co., Ltd., China); AntiGAPDH antibody (CST, \#5174); Anti-Collagen I antibody (Abcam, ab270993); AntiMMP9 antibody (Abcam, ab38898); Anti-TNF $\alpha$ antibody (CST, \#3707); Anti- $\beta$-actin antibody (CST, \#3700s); Anti-LC3B antibody (Abcam, ab192890); Anti-p- mTOR antibody (CST, \#5536); Anti-mTOR antibody (CST, \#2983); Anti-NLRP3 antibody (Proteintech, 19771-1-AP); Anti-Caspase 1 antibody (CST, \#2225); Goat anti-Mouse IgG H\&L (IRDye ${ }^{\circledR}$ 800CW) (ab216772, 1: 5000). Goat Anti-Rabbit IgG H\&L (IRDye ${ }^{\circledR}$ 680RD) preadsorbed (ab216777, 1: 5000).

\section{Animals}

Male Kunming mice(18-22g) of about 6-8 weeks were purchased from Beijing Huafukang Biotechnology Co. LTD (Certificate No. SYXK [Jing] 2019-0008, Beijing, China). Animals were maintained on a $14 \mathrm{~h} / 10 \mathrm{~h}$ light/dark cycle with temperature $20^{\circ} \mathrm{C} \pm 2^{\circ} \mathrm{C}$, and food and water were available ad libitum. The experimental procedures and animal welfare were in accordance with the Ethical Regulations on the Care and Use of Laboratory Animals of Harbin Medical University. Animals were acclimatized for three days and randomly divided into four groups ( $n=12$ per group): control group (Con), myocardial ischemia/reperfusion (MI/R) injury model group (I/R), myocardial ischemia/reperfusion (MI/R) injury + Si-Miao-Yong-An Decoction $12 \mathrm{~g} / \mathrm{kg} /$ day (SMYAD-L), and myocardial ischemia/reperfusion (MI/R) injury + Si-Miao-Yong-An Decoction $24 \mathrm{~g} / \mathrm{kg} /$ day (SMYAD-H).

\section{Establishment of Mice Myocardial Ischemia/Reperfusion (MI/R) Injury Model and Drug Treatment.}

The myocardial infarction animal model was established by left anterior descending coronary artery (LAD) ligation. Mice were anaesthetised by $2 \%$ isoflurane inhalation, 
and the skin in the operating area of mice was prepared. Endotracheal intubation was used to assist breathing and electrocardiogram was recorded. The chest was opened by a vertical cut, lateral to the left side of the sternum, the heart was temporarily exteriorized through a left thoracic incision and a 7-0 silk suture slipknot was placed around the left anterior descending coronary artery. The chest clamp was used to temporarily close the chest, and Electrocardiograph (ECG) was recorded to observe whether the ST segment was elevated. After $45 \mathrm{~min}$ of ischemia, the slipknot was released, allowing the myocardial reperfusion. Used 5-0 polypropylene sutures to close the ribcage and the muscle layer in turn. After removal of the tracheal intubation tube the anima was placed in a clean recovery cage under a heat lamp. Control group mice underwent the same operation except that the suture placed around the LAD was not tied. On the 15th day after modeling, all mice except those in the Con group and I/R group were orally treated with saline, $10 \mathrm{ml} / \mathrm{kg} / \mathrm{day}$, and the mice in the SMYAD-L group and SMYAD-L group were continuously administrated with Si-Miao-Yong-AnDecoction (at a dose of $12 \mathrm{~g} / \mathrm{kg} / \mathrm{d}$ or $24 \mathrm{~g} / \mathrm{kg} / \mathrm{d}$, corresponded to the extract $4 \mathrm{~g} / \mathrm{kg} / \mathrm{d}$ and $8 \mathrm{~g} / \mathrm{kg} / \mathrm{d}$ respectively) for 28 days, as the Figure 1 .

\section{Cardiac Functions Detected by Cardiac Magnetic Resonance (CMR)}

After 28 days of continuous administration, Cardiac Magnetic Resonance (CMR) detected cardiac function, including Left Ventricular End-Diastolic Volume (LV-EDA), Left Ventricular End-Systolic Volume (LV-ESV), LV-ligation zone thickness, Downstream thickness of LV-ligation zone and Ejection Fraction (EF). The CMR examinations were performed on a 9.4 T preclinical scanner (Bruker, BioSpec 94/20 USR TT), equipped with $660 \mathrm{mT} / \mathrm{m}$ gradients (slew-rate: $4200 \mathrm{~T} / \mathrm{m} / \mathrm{s}$ ) and three circular polarized mouse body volume coils with the inner diameter of $10 \mathrm{~mm}, 20 \mathrm{~mm}$ and 30 $\mathrm{mm}$. Mice were general anesthetized (Isoflurane, 2.5\% for induction and $1.5 \%$ for maintenance, $2 \mathrm{~L} / \mathrm{min}$ oxygen). Respiration and body temperature were monitored during CMR (SA Instruments, Inc., Stony Brook, NY, USA) and maintained at about 30 breaths-per-minute and $37^{\circ} \mathrm{C}$, respectively. CMR protocol included: pre-contrast 4 chambers long-axis and 2 chambers long-axis 2D intragate cine FLASH sequences (repetition time $=30 \mathrm{~ms}$, echo time $=2.5 \mathrm{~ms}$, number of repetitions $=1$; field of view $=38.4 \times 38.4 \mathrm{~mm}$, slice thickness $=0.8 \mathrm{~mm}$, total scan time $=1 \mathrm{~min} 36 \mathrm{~s}$ ) which were used to plan the short-axis ce-3D-IG-cine stack (repetition time $=7 \mathrm{~ms}$, echo time $=$ $2.423 \mathrm{~ms}$, number of repetitions $=1$, flip angle $=15^{\circ}$, slice thickness $=1 \mathrm{~mm}$, field of view $=20 \times 20 \mathrm{~mm}$; total scan time $=18 \mathrm{~min} 40 \mathrm{~s}$ ) encompassing the entire LV from the base to the apex.

\section{Histopathological Analysis}

Mice were sacrificed and the hearts were harvested on the 30th day after the drug treatment. $10 \%$ formaldehyde fixed the heart tissues were embedded in paraffin and later sectioned onto the glass slides $(4 \mu \mathrm{m})$. The heart tissue sections were stained with Hematoxylin \& Eosin (H\&E) to assess for myocardial I/R injury and with Masson's Trichrome staining to assess for fibrosis in the heart. 


\section{Cells Culture for H9C2 Cell Line}

H9C2 cell line was purchased from American Type Culture Collection (ATCC). H9C2 cells were maintained in complete medium (Dulbecco's modified Eagle's medium (DMEM) supplemented with $10 \%$ fetal bovine serum (FBS) and $1 \%$ penicillin/streptomycin), and incubated in a humidified $5 \% \mathrm{CO}_{2}$ incubator at $37^{\circ} \mathrm{C}$. The medium was replaced every 2-3 days, and the cells were digested with $0.25 \%(\mathrm{w} / \mathrm{v})$ Trypsin- $0.53 \mathrm{mM}$ EDTA when the density of the cells reached approximately $80-90 \%$ confluent. A subcultivation ratio of 1:2 to $1: 4$ is recommended.

\section{Establishment of Hypoxia/ Reoxygenation(H/R) Model in vitro and Drug Treatment}

When the H9C2 cells grew to approximately 70-80\% confluence, they were synchronized by fresh DMEM media without serum or antibiotics, and then the medium was removed from the synchronized H9C2 cells. H9C2 cells were inoculated into 96well plates or 6-well plates and treated for the subsequent experiments. When the density of cells reached approximately $50-60 \%$ in the plates, the Hypoxia/ Reoxygenation(H/R) model group cells were incubated $4 \mathrm{~h}$ with serum and glucose-free medium in the three-gas incubator (NUAIRE, NU-5741E, American) with a mixture of $94 \% \mathrm{~N}_{2}, 5 \% \mathrm{CO}_{2}$, and $1 \% \mathrm{O}_{2}$ at $37^{\circ} \mathrm{C}$. Subsequently, the cells were removed to the complete medium and normoxic chamber for $20 \mathrm{~h}$ to establish reoxygenation.

The experimental groups were divided into Si-Miao-Yong-An Decoction (SMYAD) group and Si-Miao-Yong-An Decoction under Hypoxia/ Reoxygenation (H/R+ SMYAD) group. At the beginning of reoxygenation, the drug was added into the completed medium according to the SMYAD concentration gradient $(0,50,75,100$, $125,150 \mu \mathrm{g} / \mathrm{mL})$.

\section{Cell Viability Assays}

Cell viability was determined by the Methyl Thiazolyl Tetrazolium (MTT) assays. Cells were seeded at $1 \times 10^{5}$ cells/well in 96-well plates overnight. After the treatment of different culture environment or drug concentration, cells were incubated with 50ul 5 $\mathrm{mg} / \mathrm{mL}$ MTT for 2-4h and subsequently solubilized in $150 \mu \mathrm{L}$ DMSO. Cell viability was assessed by measuring the absorbance at $490 \mathrm{~nm}$ by using a microtiter plate reader (Tecan, Switzerland).

\section{Enzyme-Linked Immunosorbent Assay (ELISA) for Interleukin (IL)-1ß}

Cells culture supernatants of 96-well plates were collected for assessing the concentrations of IL-1 $\beta$ in the supernatants by ELISA according to manufacturer's instructions. In briefly, the cell culture media were centrifuged at 2,000 x g for 10 minutes to remove debris. all reagents, samples, and standards were prepared as instructions. The $50 \mu \mathrm{L}$ of sample and $50 \mu \mathrm{L}$ of Antibody Cocktail were added in order to the 96-well plate, and incubated for 1 hour at room temperature on a plate shaker. Then, the 96-well plate were washed three times, added $100 \mu \mathrm{L}$ of TMB development solution and $100 \mu \mathrm{L}$ of Stop Solution, and recorded the OD at $450 \mathrm{~nm}$ by the microtiter plate reader (Tecan, Switzerland). 


\section{Western blot}

Total proteins in H9C2 cells or heart tissues were extracted by RIPA lysis buffer with $0.1 \%$ phenylmethanesulfonyl fluoride (PMSF) at indicated time points. BCA kit detected total protein concentration, and the protein concentration was homogenized. The protein samples were separated on $4-20 \%$ pre-cast gels at $150 \mathrm{~V}$ for $1 \mathrm{~h}$ and transferred on nitrocellulose filter (NC) membranes at $110-120 \mathrm{~V}$ for $50 \mathrm{~min}$. The membrane was blocked for $30 \mathrm{~min}$ at room temperature in blocking buffer to reduce non-specific binding. After blocking, the membranes were incubated with primary antibodies (Collagen I, 1:1000; MMP9, 1:1000; TNF $\alpha, 1: 1000$; LC3B, 1:1000; pmTOR, 1:1000; mTOR, 1:1000; NLRP3, 1:500; pro-Caspase1, 1:1000; cleavedCaspase 1, 1:1000; GAPDH, 1:1000; $\beta$-actin, $1: 1000)$ at $4^{\circ} \mathrm{C}$ overnight. After washing with TBS containing $0.1 \%$ Tween 20 , the membranes were followed by $1 \mathrm{~h}$ incubation with fluorescent secondary antibodies at $37^{\circ} \mathrm{C}$.

\section{Statistical Analysis}

The results were presented as the means \pm standard deviation. All analyses were performed using SPSS 19.0 statistical software. All statistics and data evaluation were subjected to statistical analysis using one-way ANOVA. ${ }^{\#} \mathrm{P}<0.05,{ }^{\# \#} \mathrm{P}<0.01,{ }^{*} \mathrm{P}<0.05$, ${ }^{* *}<0.01$, and ${ }^{* * *} \mathrm{p}<0.001$ considered significant.

\section{RESULTS}

\section{The Effect of SMYAD on Cardiac Function Index of I/R Mice Model.}

To evaluate the effect of SMYAD in I/R mice model, Cardiac Magnetic Resonance (CMR) was performed to observe the cardiac function index changes. As shown in Figure $2 \mathrm{~B}$, in the coronal view diagram of $\mathrm{CMR}$, it was found that the inner diameter of the left ventricle was significantly increased at the end of diastole and end of systole, and the ventricle was severely dilated in the I/R model group, while the left ventricular cavity was decreased in the SMYAD-L and -H group after I/R modeling. Compared with the control group, LV-EDA, LV-ESV and LV-ligation zone thickness were significantly increased, and EF and downstream thickness of LV-ligation zone were significantly decreased in the H/R group. Compared with the I/R group, both SMYAD$\mathrm{L}$ and SMYAD-H improved myocardial infarction symptoms and cardiac function to varying degrees (Figure 2A, C-F).

Figure 2. The coronal view diagram of CMR and the effect of SMYAD on cardiac functions of I/R mice model ( $\mathrm{n}=3$ ). LA: Left Atrial; LV: Left Ventricular; RV: Right Ventricular. Ventricular End-Diastolic Volume (LV-EDA), Left Ventricular EndSystolic Volume (LV-ESV), Ejection Fraction (EF). ${ }^{*} \mathrm{p}<0.05,{ }^{* *} \mathrm{p}<0.01$ vs control group, ${ }^{\#} \mathrm{p}<0.05,{ }^{\#} \mathrm{p}<0.01 \mathrm{vs} \mathrm{I} / \mathrm{R}$ group.

\section{The Effect of SMYAD on Pathological Changes of I/R Mice Model in the Heart Tissue.}

The study evaluated the changes in cardiac structure and myocardial tissue by 
histopathological staining concluding Hematoxylin \& Eosin (H\&E) and with Masson's Trichrome staining. According to HE staining, the myocardium of the control group was dyed evenly with normal myofibrils with a neat arrangement and myocardial cells were in order. In the I/R model group, the cardiac cavity of mice was significantly enlarged and the myocardial infarction area was visible. In addition, necrosis of massive myocardial cells, muscle fibers dissolution, and deposition of the cell matrix were observed. These changes of cardiac structure markedly improved in the SMYAD groups, especially the SMYAD-H group (Figure3A). According to Masson staining, the myocardial infarction and severe fibrosis were observed with increased blue scar tissue at the edge of the infarction area in the I/R model group. The myocardial infarction symptoms and fibrosis response in both SMYAD groups were alleviated (Figure3B).

Figure3. The effect of SMYAD on pathological changes of I/R mice model in the heart tissue ( $\mathrm{n}=3)$. $\mathbf{A}$ and $\mathbf{B}$ were representative images of HE staining and Masson staining in whole heart respectively. Scale bars is $1 \mathrm{~mm}$. C showed the results of the quantitative analysis of Masson staining using Image $\mathrm{J}$.

\section{The Effect of SMYAD on Expression of Collagen I, MMP9, and TNF $\alpha$ Protein in} the Heart Tissue of I/R Mice Model.

The western blot for expression of Collagen I, MMP9 and TNF $\alpha$ protein in the heart tissue of I/R mice model. As shown in Figure 4, the relative expression of each lane was normalized by the control group in the first lane. The results showed that the expression of Collagen I, MMP9, and TNF $\alpha$ protein in the model group was higher than that in the control group, and the SMYAD-H and SMYAD-L groups decreased the expression of Collagen I, MMP9, and TNF $\alpha$ protein after I/R.

Figure 4. The effect of SMYAD on expression of Collagen I, MMP9 and TNF $\alpha$ protein in the heart tissue of $\mathrm{I} / \mathrm{R}$ mice model $(\mathrm{n}=3)$. A and B were protein level of Collagen I, MMP9 and TNF $\alpha$ in each group by Western blot. ${ }^{*} p<0.05$, ${ }^{*} \mathrm{p}<0.01$ vs control group, $\# \mathrm{p}<0.05$, \#\# $\mathrm{p}<0.01 \mathrm{vs} \mathrm{I/R}$ group.

\section{The Protective Effect of SMYAD on Myocardial Cells Injured by H/R Model}

Under normal culture conditions, SMYAD did not affect the viability of H92C cells in the concentration range of $50-150 \mathrm{ug} / \mathrm{mL}$. Under hypoxia/reoxygenation model conditions, SMYAD increased cell viability in a concentration-dependent manner in the range of 50-100ug/mL (Figure 5). The results showed that SMYAD had a protective effect on cardiomyocytes injured by Hypoxia/Reoxygenation in a dose dependent way from 50 to $150 \mathrm{ug} / \mathrm{ml}$.

Figure 5. The protective effect of different doses of $\operatorname{SMYAD}(0,50,75,100,125$ and $150 \mathrm{ug} / \mathrm{mL}$ ) on the viability of cultured H9c2 cardiomyocytes under the normal and $\mathrm{H} / \mathrm{R}$ conditions $(\mathrm{n}=6)$, as determined by the MTT Assay Kit. ${ }^{*} \mathrm{p}<0.05,{ }^{* *} \mathrm{p}<0.01,{ }^{* * *}$ $\mathrm{p}<0.001 \mathrm{vs} \mathrm{H} / \mathrm{R}$ group. 


\section{The Effect of SMYAD on Autophagy of Myocardial Cells Injured by H/R Model}

Western blot analysis demonstrated the LC3B-I, LC3B-II, mTOR and p-mTOR protein levels from the total protein of cardiomyocytes, and the relative expression of each sample was normalized by the control group lane. Compared with the control group, the rate of $\mathrm{p}-\mathrm{mTOR} / \mathrm{mTOR}$ was significantly increased in the H/R group. Compared with the H/R group, the rate of p-mTOR /mTOR was significantly decreased, and LC3B-II/ LC3B-I was significantly increased in the SMYAD-L $(75 \mu \mathrm{g} / \mathrm{mL})$ and SMYAD-H $(150 \mu \mathrm{g} / \mathrm{mL})$ group (Figure 6).

Figure 6. The effect of SMYAD on autophagy of myocardial cells injured by H/R model. $\mathbf{A}$ and $\mathbf{B}$ were protein level of $\mathrm{p}-\mathrm{mTOR}, \mathrm{mTOR}$ and LC3B-I/II in each group by Western blot $(\mathrm{n}=3) .{ }^{* *} \mathrm{p}<0.01$ vs control group, ${ }^{\#} \mathrm{p}<0.05,{ }^{\#} \mathrm{p}<0.01$ vs I/R group.

\section{The Effect of SMYAD on Pyroptosis of Myocardial Cells Injured by H/R Model}

Western blot analysis demonstrated the NLRP3, pro-Caspase 1 and cleaved-Caspase 1 protein levels and ELISA analysis detected the expression level of IL-1 $\beta$. Compared with the control group, the expression of NLRP3, pro-Caspase 1 and cleaved-Caspase 1 were significantly increased in the $H / R$ group. Compared with the $H / R$ group, the expression of NLRP3, pro-Caspase 1 and cleaved- Caspase 1 was decreased in the SMYAD-L $(75 \mu \mathrm{g} / \mathrm{mL})$ and SMYAD-H $(150 \mu \mathrm{g} / \mathrm{mL})$ group (Figure 7$)$.

Figure 7. The effect of SMYAD on pyroptosis of myocardial cells injured by H/R model. $\mathbf{A}$ and $\mathbf{B}$ were protein level of NLRP3, pro-Caspase 1 and cleaved-Caspase 1 in each group by Western blot $(n=3)$. $C$ was expression level of IL-1 $\beta$ by in each group ELISA. ${ }^{* *} \mathrm{p}<0.01$ vs control group, ${ }^{*} \mathrm{p}<0.05 \mathrm{vs} \mathrm{I/R}$ group.

\section{DISCUSSION}

MI is characterized by massive myocardial cell death and has a highly likely poor prognosis [21]. Although prevention and treatment works have been greatly improved, myocardial infarction still has a significant impact on global health and is also the main cause of mortality worldwide[22]. The therapeutic regimes for MI contain thrombolytic therapy, coronary intervention therapy, and drug therapy. All approaches are aimed at restoring blood supply to the ischemic zone and replenishing nutrients and oxygen [23, 24]. However, concerns have been raised about the lack of treatments on the subsequent $\mathrm{I} / \mathrm{R}$ injury $[25,26]$.

Accumulating evidence has proved that SMYAD can improve heart function and alleviate cardiac fibrosis in the mice models of heart failure [19, 20, 27]. In this study, a mouse MI model induced by I/R injury was performed to evaluate the cardioprotective effects of SMYAD. After 4 weeks of treatment, the functional test for mice hearts was performed by CMR. We found there was not much difference in EDV among model and SMYAD groups, but the difference in ESV among them was significant. Moreover, the left ventricular wall downstream to the ligation position was thicker in the mice of 
SMYAD groups than that in the Model group, and the EF of left ventricle in the mice of SMYAD group was also higher than that in the Model group (Figure 2). All these results suggested that 4 weeks of application of SMYAD effectively maintained the cardiac functions of I/R injury by the inhibition of cardiac remodeling and the reduction of myocardiocytes loss. The following H\&E and Masson staining indicated that 4 weeks of application of SMYAD reduced the infarct sizes and inhibited the myocardial fibrosis (Figure 3). When MI occurs, inflammation mediates myocardial fibrosis [28] and the abnormal proliferation of collagen in the myocardial interstitium [29], which seriously affects cardiac function. Matrix metalloproteinases (MMPs) act as targets of myocardial fibrosis to affect the process of myocardial fibrosis[30]. SMYAD reduced the expression of heart failure markers and fibrosis markers (Collagen I, MMP9, and $\mathrm{TNF} \alpha$ ) in the heart tissue of model animals after myocardial infarction (Figure 4). To prove the hypothesis that the therapeutic effects of SMYAD in MI were partially dependent on the protective capabilities of cardiomyocytes. The in vitro [31,32] study of oxygen-glucose deprivation (OGD) in H29C cardiomyocytes was performed to mimic the H/R injury model. The results indicated that SMYAD protected myocardial cells from OGD in a dose-dependent way (Figure 5).

The mechanisms of Myocardial I/R injury are intricate, including calcium overload, oxygen free radicals, and inflammatory response, which eventually triggers the process of PCD in hearts [33-36]. Pyroptosis, as one of PCD forms, is called inflammatory cell death and mainly depends on the formation of inflammasome NLRP3 that activates the Caspase-1 to release the IL-1 $\beta$ and IL-18 into the extracellular environment, recruiting inflammatory cells to aggregate the inflammatory response [10, 11]. We found that SMYAD reduced the expressions levels of NLRP3 and Caspase-1 in the OGD of H9C2 cardiomyocytes. The concentration of IL-1 $\beta$ in the culture supernatant among groups was detected by ELISA, and SMYAD reduced the IL- $1 \beta$ yield in a dose-dependent way. Have demonstrated that SMYAD targeted and inhibited on the pyroptosis pathway, we next sought to identify the counterbalancing mechanism. When myocardial I/R injury occurs, autophagy will be activated. On the one hand, autophagy helps to clear damaged organelles and proteins, on the other hand provides energy for ischemic cells [37, 38]. Studies have shown that maintaining autophagy flux during ischemia-reperfusion can reduce infarct size and protect cardiomyocytes $[39,40]$. The mTOR signaling pathway is considered an important signaling pathway during cardiac I/R injury. Studies have shown that the decrease of phosphorylated mTOR level may lead to the activation of autophagy [41]. The change of microtubule-associated protein 1A/1B-light chain 3B (LC3B-II) is a well-established marker of autophagosome formation. In this study, we found that SMYAD elevated the protein expression of LC3B-II and the rate of $\mathrm{p}$ $\mathrm{mTOR} / \mathrm{mTOR}$ in the OGD of H29C cardiomyocytes, which suggested that the antipyroptosis of SMYAD resulted from the activation of endogenous autophagy.

\section{CONCLUSIONS}

In this study, SMYAD protected myocardial tissue and improved cardiac function in the mouse MI model induced by $\mathrm{I} / \mathrm{R}$ injury. Moreover, SMYAD exerted effects on 
cardiomyocyte protection by activating autophagy and inhibiting pyroptosis in vitro. These findings support the clinical application of SMYAD for patients suffered from MI, especially in preventing the myocardial tissue from I/R injury.

\begin{abstract}
ABBREVIATIONS
SMYAD : Si-Miao-Yong-An decoction; CMR: Cardiac Magnetic Resonance; H/R : hypoxia/reoxygenation; MI: Myocardial infarction; IR: ischemia/reperfusion; PCD: Programmed cell death; DAMPs: damage-associated molecular patterns; DMEM: Dulbecco's modified Eagle's medium; LAD: left anterior descending coronary artery; MI/R : Myocardial Ischemia/Reperfusion; ECG :Electrocardiograph; HE: Hematoxylin \& Eosin; ATCC: American Type Culture Collection; FBS: fetal bovine serum; MTT: Methyl Thiazolyl Tetrazolium; ELISA Enzyme-Linked Immunosorbent Assay; IL: Interleukin; PMSF: phenylmethanesulfonyl fluoride; NC: nitrocellulose filter; LV-EDA: Left Ventricular End-Diastolic Volume; LV-ESV: Left Ventricular End-Systolic Volume; $\mathrm{EF}$ :Ejection Fraction; OGD : oxygen-glucose deprivation.
\end{abstract}

\title{
DATA AVAILABILITY STATEMENT
}

All datasets generated for this study are included in the manuscript file.

\section{ETHICS STATEMENT}

The experimental procedures and animal welfare were in accordance with the Ethical Regulations on the Care and Use of Laboratory Animals of Harbin Medical University.

\section{ACKNOWLEDGEMENTS}

Not applicable.

\section{DISCLOSURE}

All authors have no financial, personal, or other relationships with other people or organizations that could inappropriately influence the work.

\section{AUTHOR CONTRIBUTIONS}

YH, YL, XS and WC conceived and designed the experiments. WC, LZ, MW, YH, and YZ carried out the experiments, analyzed the data, and drafted the manuscript. WC, XS, LZ, MW and YH participated in its design and prepared the paper. WC, XS, YH, and YL revised the manuscript. All authors read and approved the final manuscript.

\section{CONSENT FOR PUBLICATION}

We confirm that the manuscript has been read and approved for publication by all named authors and that there are no other persons who satisfied the criteria for authorship but are not listed.

\section{FUNDING}

This study was supported by the China Postdoctoral Science Foundation (Grant No. 2016M602697, No. 2018T110988). 


\section{REFERENCES}

1. Thygesen, K., et al., Universal definition of myocardial infarction. J Am Coll Cardiol, 2007. 50(22): p. 2173-95.

2. Binder, A., et al., Myocardial protection from ischemia-reperfusion injury post coronary revascularization. Expert Rev Cardiovasc Ther, 2015. 13(9): p. 1045-57.

3. Weintraub, W.S., et al., Value of primordial and primary prevention for cardiovascular disease: a policy statement from the American Heart Association. Circulation, 2011. 124(8): p. 967-90.

4. Tower, J., Programmed cell death in aging. Ageing Res Rev, 2015. 23(Pt A): p. 90-100.

5. Nagata, S. and M. Tanaka, Programmed cell death and the immune system. Nat Rev Immunol, 2017. 17(5): p. 333-340.

6. D'Arcy, M.S., Cell death: a review of the major forms of apoptosis, necrosis and autophagy. Cell Biol Int, 2019. 43(6): p. 582-592.

7. Yue, R.C., et al., Calpain silencing alleviates myocardial ischemia-reperfusion injury through the NLRP3/ASC/Caspase-1 axis in mice. Life Sci, 2019. 233: p. 116631.

8. Kurian, G.A., et al., Antioxidant effects of ethyl acetate extract of Desmodium gangeticum root on myocardial ischemia reperfusion injury in rat hearts. Chin Med, 2010. 5: p. 3.

9. Rauf, A., et al., Role of Caspase 1 in Ischemia/Reperfusion Injury of the Myocardium. J Cardiovasc Pharmacol, 2019. 74(3): p. 194-200.

10. $\mathrm{Xu}, \mathrm{Y} . \mathrm{J} .$, et al., Pyroptosis and its relationship to atherosclerosis. Clin Chim Acta, 2018. 476: p. 28-37.

11. Zhaolin, Z., et al., Role of pyroptosis in cardiovascular disease. Cell Prolif, 2019. 52(2): p. e12563.

12. Takahashi, M., Cell-Specific Roles of NLRP3 Inflammasome in Myocardial Infarction. J Cardiovasc Pharmacol, 2019. 74(3): p. 188-193.

13. Yao, W., et al., Oxidative Stress and Inflammation Interaction in Ischemia Reperfusion Injury: Role of Programmed Cell Death. Oxid Med Cell Longev, 2019. 2019: p. 6780816.

14. Wu, M.Y., et al., Current Mechanistic Concepts in Ischemia and Reperfusion Injury. Cell Physiol Biochem, 2018. 46(4): p. 1650-1667.

15. Lee, M.S., Role of mitochondrial function in cell death and body metabolism. Front Biosci (Landmark Ed), 2016. 21: p. 1233-44.

16. Sciarretta, S., et al., The Role of Autophagy in the Heart. Annu Rev Physiol, 2018. 80: p. 1 26.

17. Zhao, Y., et al., Dissection of mechanisms of Chinese medicinal formula Si-Miao-Yongan decoction protects against cardiac hypertrophy and fibrosis in isoprenaline-induced heart failure. J Ethnopharmacol, 2020. 248: p. 112050.

18. Liu, Z., et al., Promotion of classic neutral bile acids synthesis pathway is responsible for cholesterol-lowing effect of Si-miao-yong-an decoction: Application of LC-MS/MS method to determine 6 major bile acids in rat liver and plasma. J Pharm Biomed Anal, 2017. 135: p. 167-175.

19. Ren, Y., et al., Si-Miao-Yong-An decoction ameliorates cardiac function through restoring the equilibrium of SOD and NOX2 in heart failure mice. Pharmacol Res, 2019. 146: p. 104318. 
20. Su, C., et al., Si-Miao-Yong-An Decoction Protects Against Cardiac Hypertrophy and Dysfunction by Inhibiting Platelet Aggregation and Activation. Front Pharmacol, 2019. 10: p. 990.

21. McCarthy, C.P., J.L. Januzzi, Jr., and H.K. Gaggin, Type 2 Myocardial Infarction- An Evolving Entity. Circ J, 2018. 82(2): p. 309-315.

22. Kartalis, A., et al., Trends in acute myocardial infarction incidence and relative outcomes on a certain insular Mediterranean population. Int J Cardiol, 2014. 173(2): p. 320-1.

23. Mendoza-Torres, E., et al., Protection of the myocardium against ischemia/reperfusion injury by angiotensin-(1-9) through an AT2R and Akt-dependent mechanism. Pharmacol Res, 2018. 135: p. 112-121.

24. Russo, l., et al., Platelets, diabetes and myocardial ischemia/reperfusion injury. Cardiovasc Diabetol, 2017. 16(1): p. 71.

25. Grek, A., et al., Sepsis and Shock Response Team: Impact of a Multidisciplinary Approach to Implementing Surviving Sepsis Campaign Guidelines and Surviving the Process. Am J Med Qual, 2017. 32(5): p. 500-507.

26. Zuo, Y., et al., Atorvastatin Protects Myocardium Against Ischemia-Reperfusion Injury Through Inhibiting miR-199a-5p. Cell Physiol Biochem, 2016. 39(3): p. 1021-30.

27. Zhao, Y., et al., Si-Miao-Yong-An Decoction attenuates isoprenaline-induced myocardial fibrosis in AMPK-driven Akt/mTOR and TGF-beta/SMAD3 pathways. Biomed Pharmacother, 2020. 130: p. 110522.

28. Lu, Q.Y., et al., Carthamin Yellow Protects the Heart Against Ischemia/Reperfusion Injury With Reduced Reactive Oxygen Species Release and Inflammatory Response. J Cardiovasc Pharmacol, 2019. 74(3): p. 228-234.

29. Gonzalez, A., et al., The complex dynamics of myocardial interstitial fibrosis in heart failure. Focus on collagen cross-linking. Biochim Biophys Acta Mol Cell Res, 2019. 1866(9): p. 1421-1432.

30. Chaturvedi, P., et al., Cardiosome mediated regulation of MMP9 in diabetic heart: role of mir29b and mir455 in exercise. J Cell Mol Med, 2015. 19(9): p. 2153-61.

31. Chen, Y., et al., Pretreatment of ghrelin protects H9c2 cells against hypoxia/reoxygenation-induced cell death via PISK/AKT and AMPK pathways. Artif Cells Nanomed Biotechnol, 2019. 47(1): p. 2179-2187.

32. Ren, Z., et al., Fibroblast growth factor-21 alleviates hypoxia/reoxygenation injury in H9c2 cardiomyocytes by promoting autophagic flux. Int J Mol Med, 2019. 43(3): p. 1321-1330.

33. Frank, A., et al., Myocardial ischemia reperfusion injury: from basic science to clinical bedside. Semin Cardiothorac Vasc Anesth, 2012. 16(3): p. 123-32.

34. Kitazume-Taneike, R., et al., Ablation of Toll-like receptor 9 attenuates myocardial ischemia/reperfusion injury in mice. Biochem Biophys Res Commun, 2019. 515(3): p. 442447.

35. Li, J.P., et al., [Relationship between calcium overload and myocardial ischemia reperfusion injury and intervention strategy of Chinese herbal medicine]. Zhongguo Zhong Yao Za Zhi, 2016. 41(11): p. 2168-2173.

36. Kalogeris, T., et al., Ischemia/Reperfusion. Compr Physiol, 2016. 7(1): p. 113-170.

37. Saito, T. and J. Sadoshima, Molecular mechanisms of mitochondrial autophagy/mitophagy in the heart. Circ Res, 2015. 116(8): p. 1477-90. 
38. Li, L., et al., The role of autophagy in cardiac hypertrophy. Acta Biochim Biophys Sin (Shanghai), 2016. 48(6): p. 491-500.

39. Zheng, Y., et al., Berbamine postconditioning protects the heart from ischemia/reperfusion injury through modulation of autophagy. Cell Death Dis, 2017. 8(2): p. e2577.

40. Bhuiyan, M.S., et al., Enhanced autophagy ameliorates cardiac proteinopathy. J Clin Invest, 2013. 123(12): p. 5284-97.

41. Inoki, K., J. Kim, and K.L. Guan, AMPK and mTOR in cellular energy homeostasis and drug targets. Annu Rev Pharmacol Toxicol, 2012. 52: p. 381-400. 


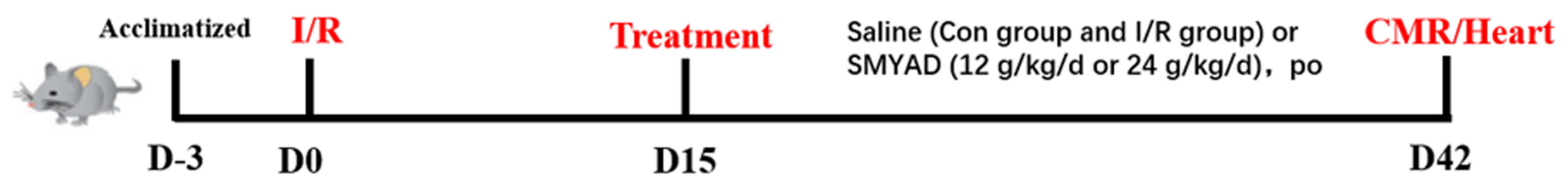

Figure 1

On the 15th day after modeling, all mice except those in the Con group and I/R group were orally treated with saline, $10 \mathrm{ml} / \mathrm{kg} /$ day, and the mice in the SMYAD-L group and SMYAD-L group were continuously administrated with Si-Miao-Yong-An-Decoction (at a dose of $12 \mathrm{~g} / \mathrm{kg} / \mathrm{d}$ or $24 \mathrm{~g} / \mathrm{kg} / \mathrm{d}$, corresponded to the extract $4 \mathrm{~g} / \mathrm{kg} / \mathrm{d}$ and $8 \mathrm{~g} / \mathrm{kg} / \mathrm{d}$ respectively) for 28 days, as the Figure 1 .
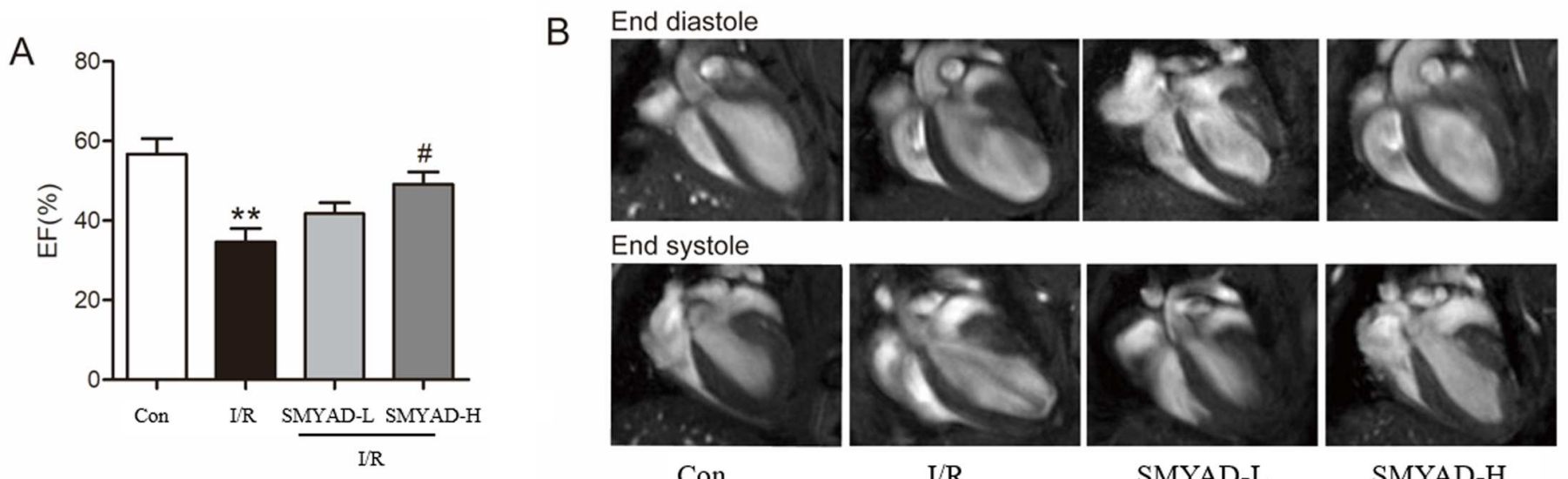

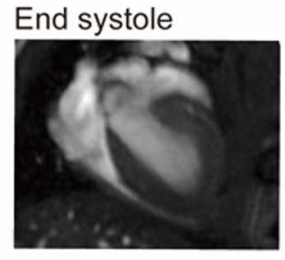

Con

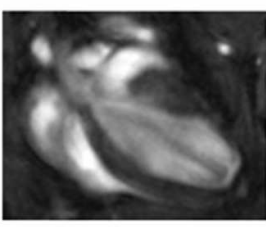

I/R

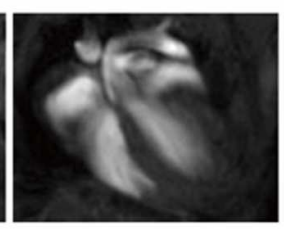

SMYAD-L

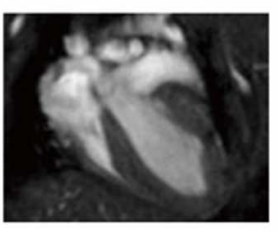

SMYAD-H
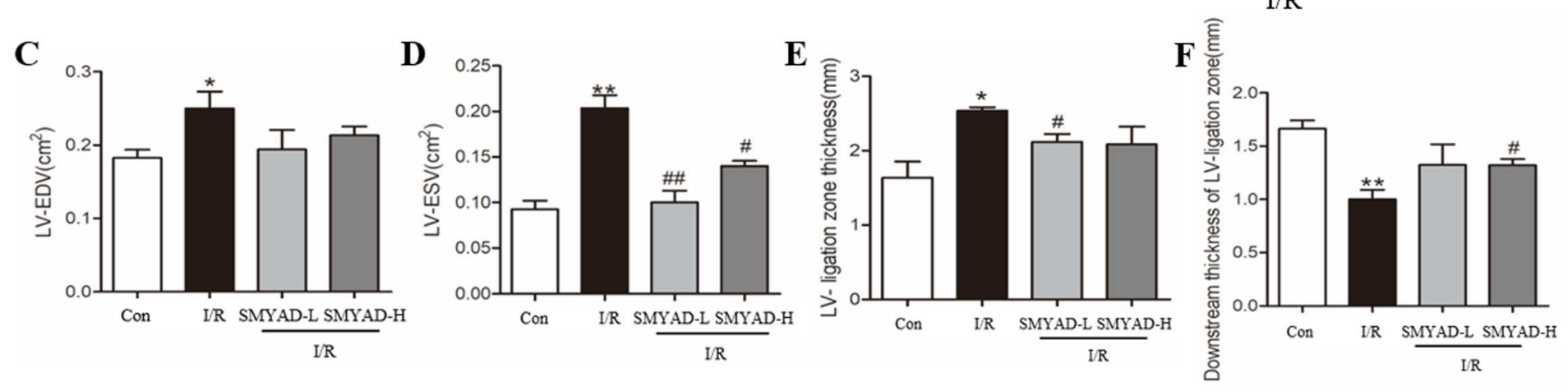

Figure 2

The coronal view diagram of CMR and the effect of SMYAD on cardiac functions of I/R mice model $(n=3)$. LA: Left Atrial; LV: Left Ventricular; RV: Right Ventricular. Ventricular End-Diastolic Volume (LVEDA), Left Ventricular End-Systolic Volume (LV-ESV), Ejection Fraction (EF). ${ }^{*} p<0.05,{ }^{*} p<0.01$ vs control group, $\# p<0.05$, \#\# $p<0.01 v$ s I/R group. 
A

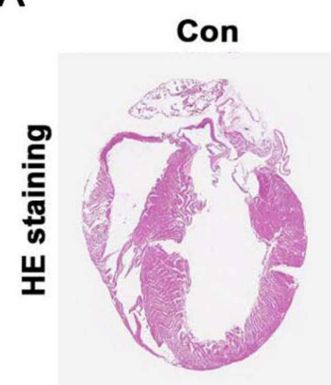

B

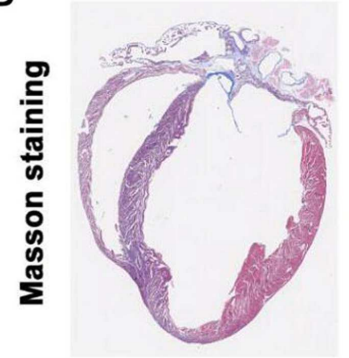

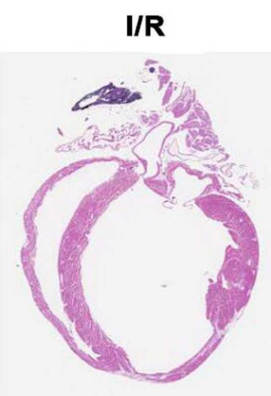

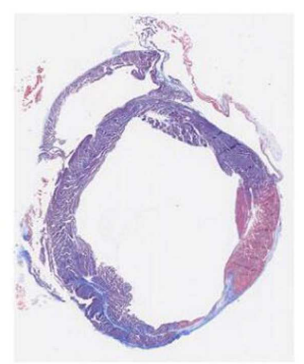

I/R
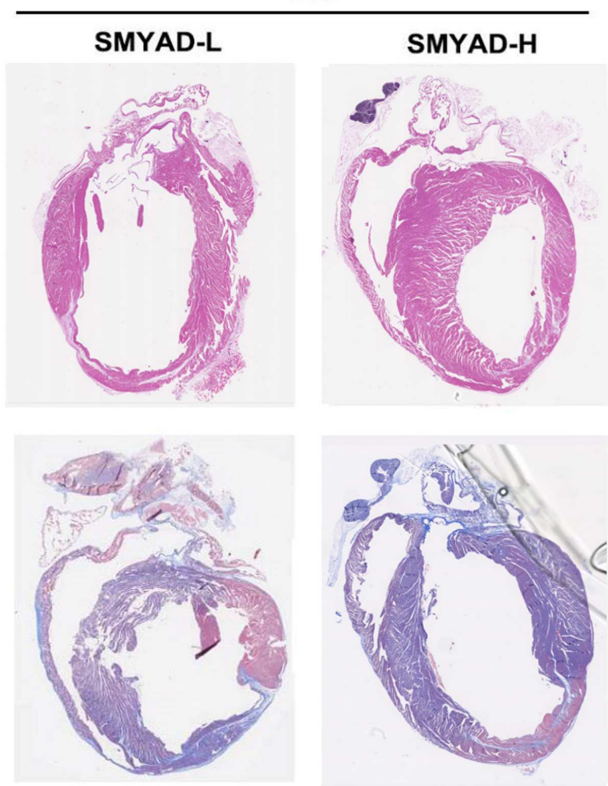

C

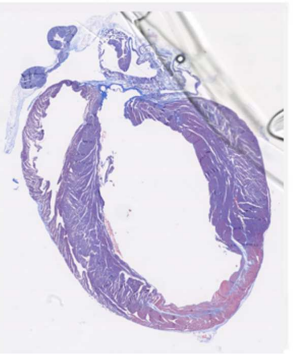

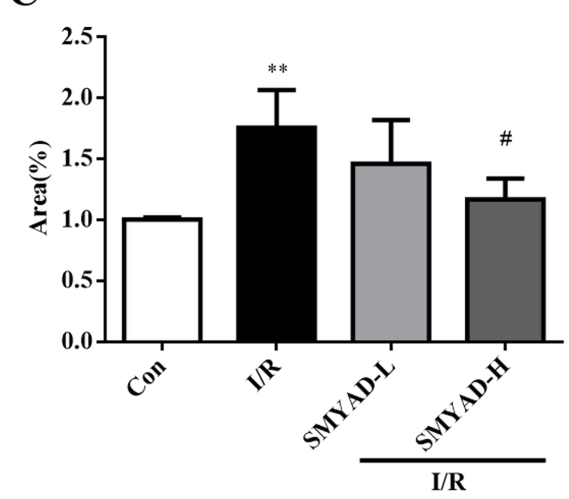

Figure 3

The effect of SMYAD on pathological changes of $I / R$ mice model in the heart tissue $(n=3)$. A and B were representative images of HE staining and Masson staining in whole heart respectively. Scale bars is $1 \mathrm{~mm}$. C showed the results of the quantitative analysis of Masson staining using Image $\mathrm{J}$. 


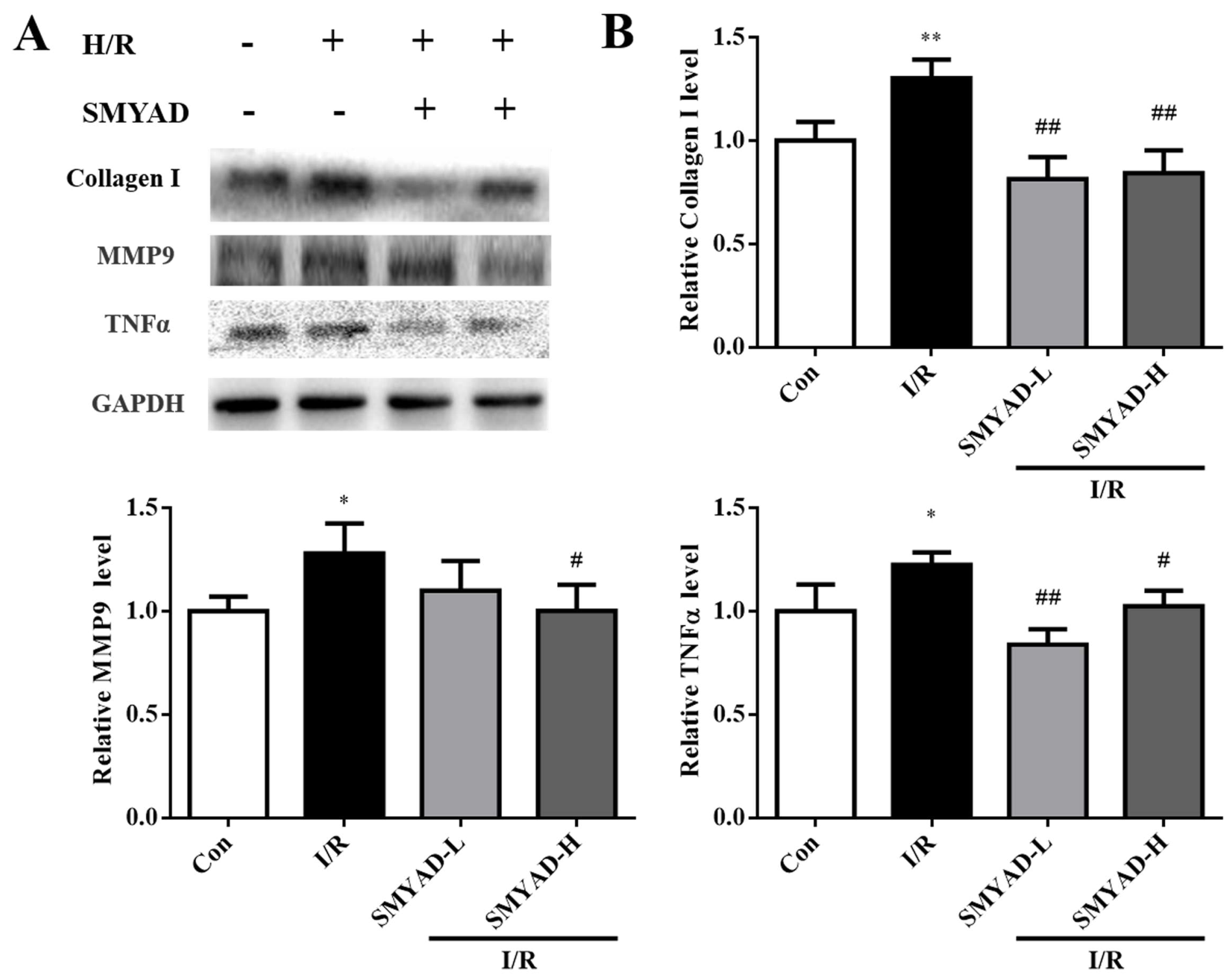

Figure 4

The effect of SMYAD on expression of Collagen I, MMP9 and TNFa protein in the heart tissue of I/R mice model $(n=3)$. A and B were protein level of Collagen I, MMP9 and TNFa in each group by Western blot. ${ }^{*} p<0.05,{ }^{*} p<0.01$ vs control group, $\# p<0.05$, \#\# $p<0.01$ vs I/R group. 


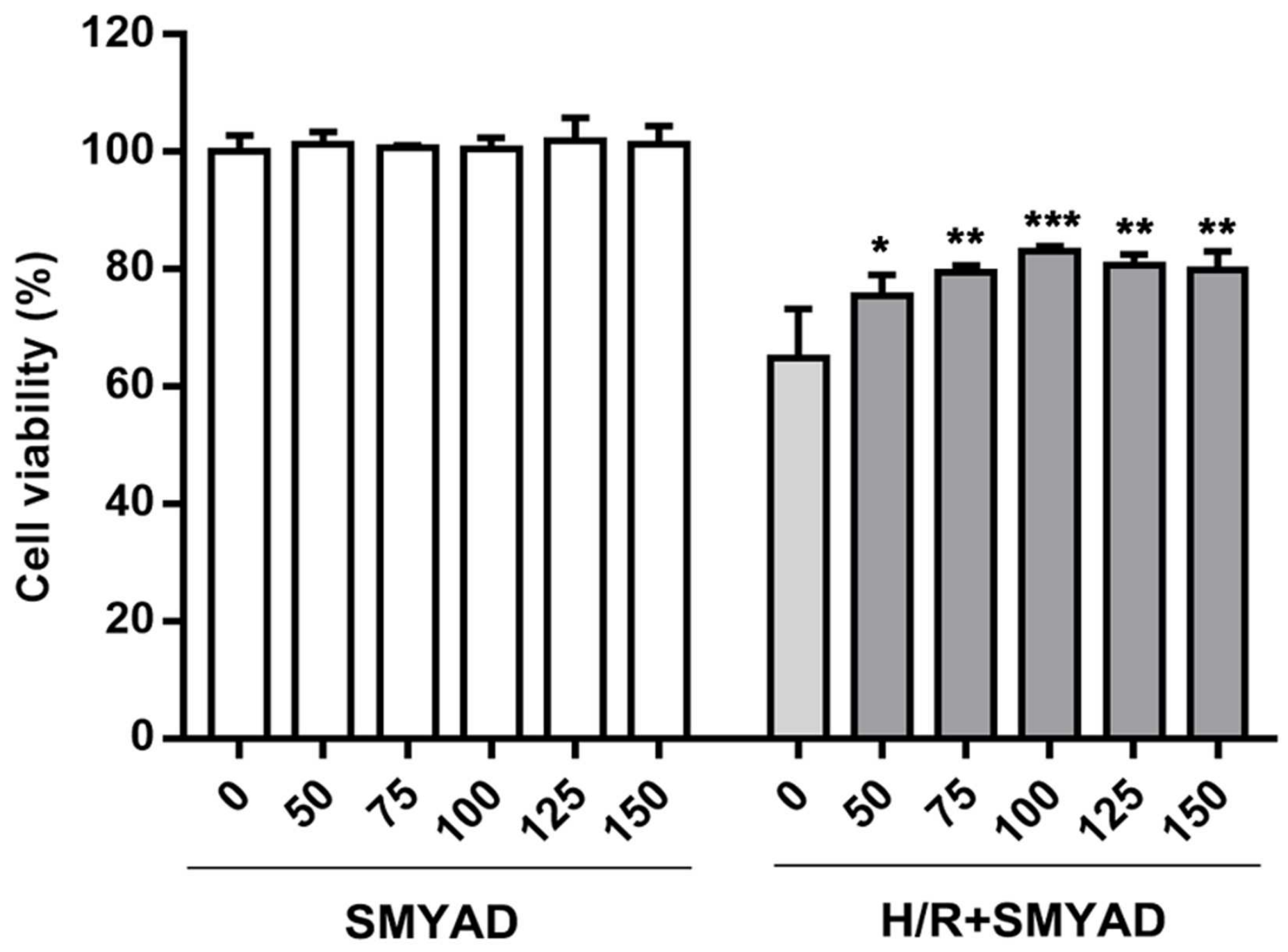

Figure 5

The protective effect of different doses of $\operatorname{SMYAD}(0,50,75,100,125$ and $150 \mathrm{ug} / \mathrm{mL})$ on the viability of cultured $\mathrm{H} 9 \mathrm{c} 2$ cardiomyocytes under the normal and $\mathrm{H} / \mathrm{R}$ conditions $(n=6)$, as determined by the MTT Assay Kit. * $p<0.05, * * p<0.01, * \star * p<0.001$ vs H/R group.

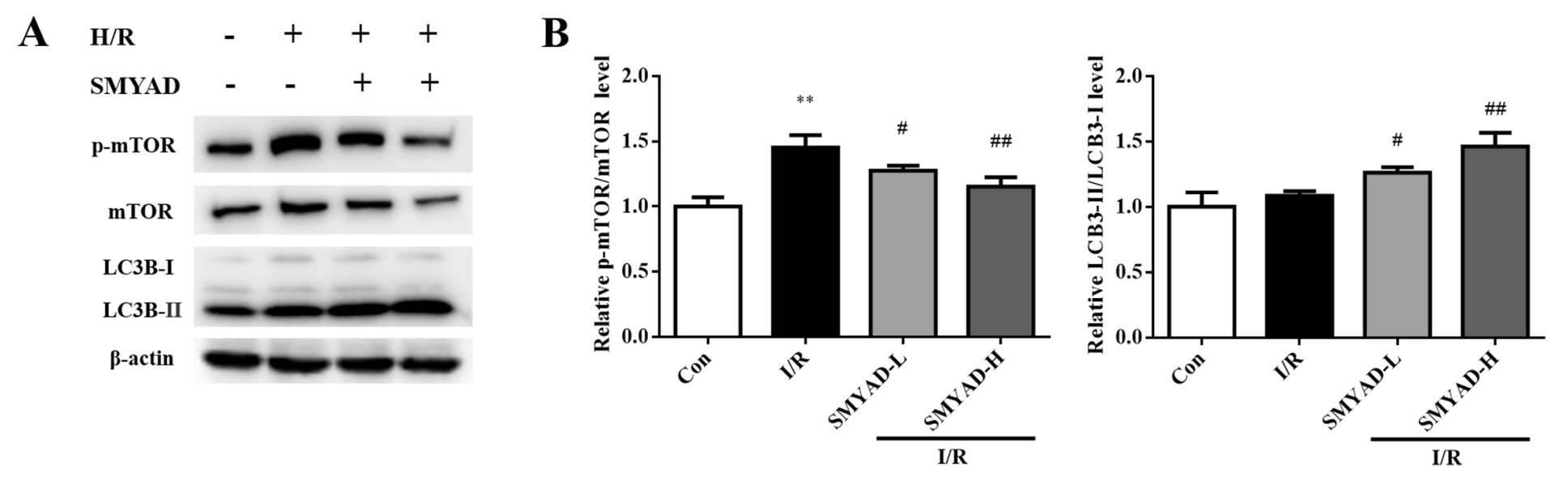


Figure 6

The effect of SMYAD on autophagy of myocardial cells injured by $H / R$ model. A and $B$ were protein level of $p$-mTOR, mTOR and LC3B囚I/II in each group by Western blot $(n=3)$. ${ }^{*} p<0.01$ vs control group, $\# p<$ 0.05 , \#\# $p<0.01$ vs I/R group.

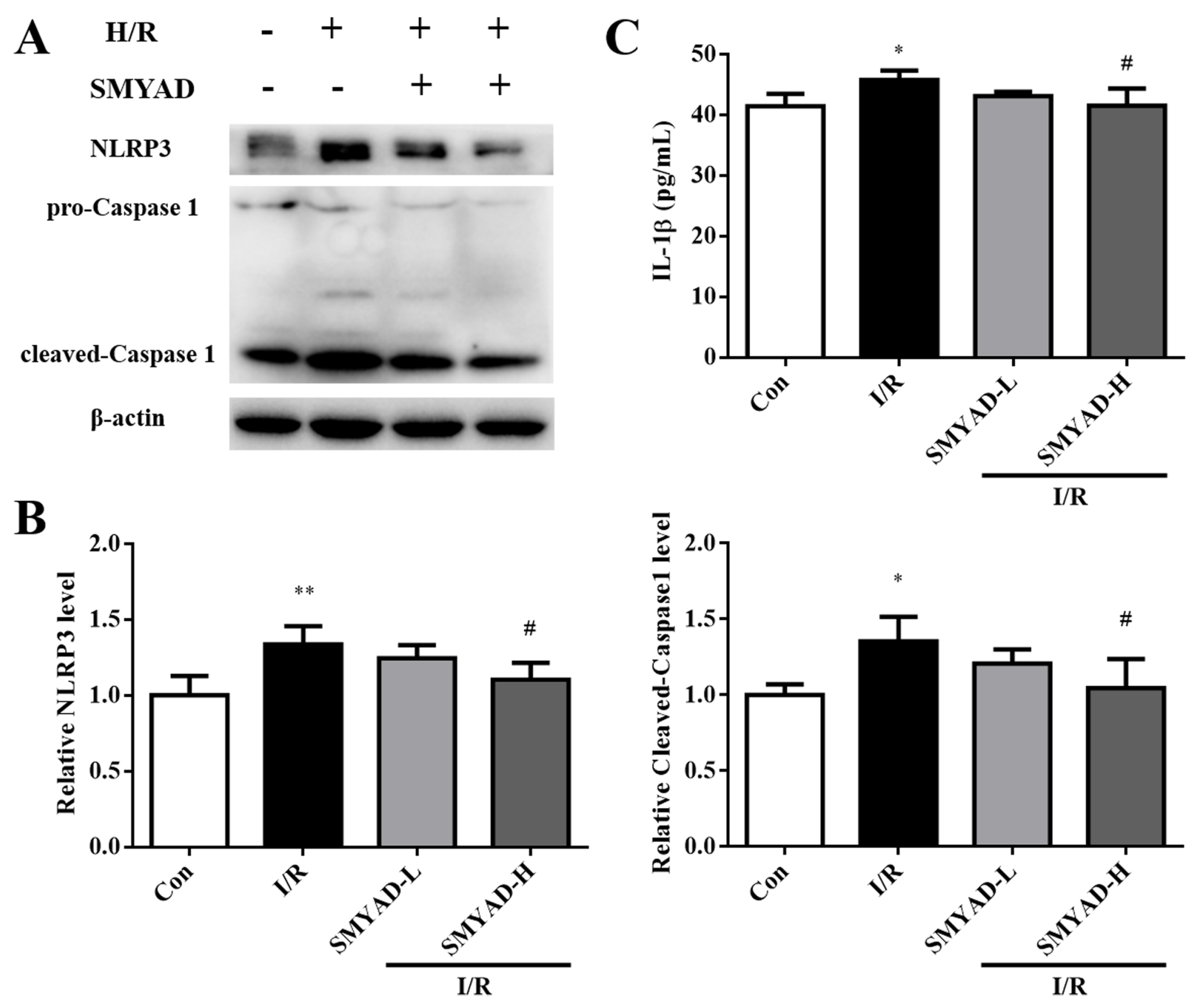

Figure 7

The effect of SMYAD on pyroptosis of myocardial cells injured by $\mathrm{H} / \mathrm{R}$ model. $A$ and $B$ were protein level of NLRP3, pro-Caspase 1 and cleaved- Caspase 1 in each group by Western blot $(n=3)$. $C$ was expression level of IL-1 $\beta$ by in each group ELISA. ${ }^{*} p<0.01$ vs control group, $\# p<0.05$ vs I/R group. 